\title{
Real-World Use of Fostamatinib in Patients with Immune Thrombocytopenia and Thrombotic Risk
}

\author{
Amit R. Mehta ${ }^{a} b$ Aron Kefelac Charina Toste ${ }^{d}$ Donald Sweet ${ }^{e}{ }^{e}$

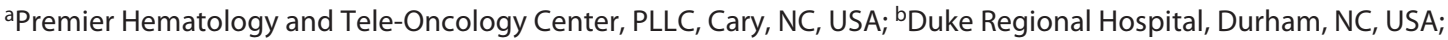 \\ ${ }^{\mathrm{C}}$ Northwest Georgia Oncology Centers, Athens, GA, USA; ${ }^{\mathrm{d} O p t u m C a r e}$ Cancer Care, Las Vegas, NV, USA; ${ }^{\mathrm{e} A M I T A}$ \\ Health Medical Group, Chicago, IL, USA; fNorthwestern University, Chicago, IL, USA
}

\section{Keywords}

Fostamatinib - Immune thrombocytopenia - Thrombosis · Cardiovascular disease

\begin{abstract}
Patients with immune thrombocytopenia (ITP) are at increased risk for bleeding and are paradoxically at increased risk for thrombosis. Many patients with ITP have underlying cardiovascular (CV) disease and/or other thrombotic risk factors for which considerable attention to selecting a therapeutic agent to manage ITP is needed. Fostamatinib, a spleen tyrosine kinase inhibitor, may reduce the risk of thrombosis while not interfering with hemostasis. We present a case series of 5 patients with ITP who had significant CV histories; each had at least 2 thrombotic risk factors. After unsuccessful management of ITP with other treatments, fostamatinib was initiated, was observed to be tolerable, and provided a durable platelet response without associated thromboembolic events. Fostamatinib may be the treatment of choice for patients with ITP in whom use of prothrombotic treatments should be avoided and/or continued use of antiplatelet or anticoagulant medication is needed.
\end{abstract}

(c) 2021 The Author(s)

Published by S. Karger AG, Basel

karger@karger.com www.karger.com/aha

Karger $\stackrel{\text { ' }}{=}$
(C) 2021 The Author(s)

Published by S. Karger AG, Basel

This is an Open Access article licensed under the Creative Common Attribution-NonCommercial-4.0 International License (CC BY-NC) (http://www.karger.com/Services/OpenAccessLicense), applicable to the online version of the article only. Usage and distribution for commercial purposes requires written permission.

\section{Introduction}

Immune thrombocytopenia (ITP) is a heterogeneous, autoimmune-mediated disorder characterized by platelet destruction, impaired platelet production, and subsequent increased risk for bleeding [1,2]. Various treatment options are available for managing ITP, each with its own set of advantages and disadvantages. Corticosteroids are typically recommended as first-line therapy, along with intravenous immunoglobulin (IVIg) and anti-D immunoglobulin. All are commonly used as rescue therapies. Subsequent medical therapy includes the use of fostamatinib, thrombopoietin-receptor agonists (TPO-RAs), or anti-CD20 monoclonal antibody; other interventions include splenectomy and, less commonly, immunosuppressive medications [3]. Unfortunately, the sequence for prescribing these subsequent-line agents is unclear due to limited evidence from randomized controlled trials, but ultimately, the choice of treatment should be individualized for each patient. When selecting an agent, a patient's comorbidities, preferences, and risk for adverse effects should be considered, and shared decision-making between the patient and provider is encouraged $[3,4]$.

Correspondence to:

Amit R. Mehta, amitmehta@ premierheme.com 
Evidence suggests that ITP inherently increases the risk for thrombosis $[2,5,6]$, and patients may have comorbidities that also increase their risk for thrombosis (e.g., obesity, age, and smoking), making certain ITP treatment options less favorable $[5,7,8]$. Specifically, use of TPO-RAs or splenectomy may be a less preferable approach for ITP patients with underlying prothrombotic conditions such as cardiovascular (CV) disease or metabolic syndrome. In the real-world setting, ITP is not always an isolated disease. Although the ultimate treatment goal should be to minimize and/or prevent bleeding episodes, this can prove to be particularly challenging when patients require treatment with an anticoagulant or antiplatelet medication [9].

Fostamatinib, a spleen tyrosine kinase (SYK) inhibitor that was approved by the FDA in April 2018, offers management of ITP through a novel mechanism of action. FC $\gamma$ receptors on the surface of macrophages detect and bind antibody-coated platelets, which trigger an SYK-dependent phosphorylation cascade, driving phagocytosis of the platelet $[10,11]$. SYK inhibition prevents phagocytosis, destruction of platelets, and subsequent events, thus interrupting disease progression $[12,13]$. Further, it has been shown that SYK plays a key role in signaling through the platelet receptors glycoprotein VI (GPVI) and C-type lectin-like receptor-2 (CLEC-2) [14-16]. Both receptors are important in thrombus formation but are dispensable for hemostasis [16-19]. Consequently, fostamatinib may reduce the risk of thrombosis by inhibiting GPVI-- and CLEC-2-mediated thrombus formation while not interfering with regular hemostasis $[14,18,20,21]$.

Fostamatinib has demonstrated efficacy in 2 phase 3 clinical trials of ITP and in an open-label extension study $[22,23]$. In a recently published post hoc analysis, fostamatinib was shown to be more effective when used in an earlier line of therapy, as a second- or third-line agent, than when introduced in later lines of therapy [24]. Fostamatinib is a twice-daily (BID) oral agent that may be given without regard to meals. It offers a tolerable safety profile, with adverse effects of diarrhea, hypertension, nausea, and increased transaminases most commonly reported in clinical studies, most of which were mild to moderate in severity and were adequately managed with dose reduction, dose interruption, or supportive care [22, 23].

We present a case series of 5 patients with ITP identified through a retrospective chart review by each author in his or her respective practice. Patients were treated with fostamatinib and, prior to ITP therapy, were considered to have an ongoing substantial clinical risk of thrombosis due to the presence of CV comorbidity, thrombophilia, or history of thrombosis with increased risk of recurrent thromboembolism. These patients initiated fostamatinib after lack of platelet response to first-line or subsequent therapies and/or with susceptibility to adverse effects, which precluded the use of other ITP treatments.

\section{Case Series}

The following cases were identified retrospectively and represent the successful experience of prescribing fostamatinib for ITP therapy in patients with concomitant CV disease and/or a history of thromboembolism. In these cases, hematologists and clinicians opted to switch patients to fostamatinib, with the rationale that this was a reasonable and safe option for a given patient who had previously tried 1 or more other therapies unsuccessfully. Table 1 highlights patient demographic information, thrombotic risk factors, and ITP characteristics. Figure 1 presents platelet response over time on fostamatinib treatment.

\section{Case 1}

A 63-year-old non-Hispanic white male was referred to a hematologist after he was diagnosed with chronic ITP by his primary care physician. He complained of mild fatigue, epistaxis, and easy bruising and presented with a platelet count of $61,000 / \mu \mathrm{L}$. Of significance, his past medical history included hypertension, hyperlipidemia, type 2 diabetes mellitus (on insulin), and unprovoked deep vein thrombosis (DVT) 4 years before ITP diagnosis. Prior detailed thrombophilia evaluation was unrevealing for an etiology of the DVT. His platelets, which had been documented as stable at over $200,000 / \mu \mathrm{L}$ for multiple years, abruptly decreased to $43,000 / \mu \mathrm{L}$. Evaluation by the hematologist was consistent with ITP, and the patient was started on oral dexamethasone $40 \mathrm{mg}$ daily for 4 days. He exhibited no meaningful response, with platelets remaining at around $40,000 / \mu \mathrm{L}$, and he complained of sleep disturbances and headaches while taking dexamethasone. In parallel with this lack of response to corticosteroids, he developed angina, and an abnormal stress test required that he undergo an elective cardiac catheterization. The cardiologist anticipated the need for coronary stenting and subsequent antiplatelet therapy. Therefore, to balance the need for coronary intervention and a falling platelet count, the patient's ITP needed to be stabilized. Upon review of treatment options for managing his ITP, and with consideration of patient preferences including for the oral route of administration, it was agreed that fostamatinib, initiated at $100 \mathrm{mg}$ PO BID, would be the next treatment. The patient responded rapidly, with platelet count rising to over $90,000 /$ $\mu \mathrm{L}$ within 2 weeks; furthermore, the patient has remained on this regimen with steady and durable increases in platelets before and after cardiac catheterization. Despite needing ongoing antiplatelet therapy with aspirin after his cardiac procedure, the patient's platelets have remained above $70,000 / \mu \mathrm{L}$ and he has not needed to increase the fostamatinib dose. Additionally, there have been no reported adverse effects requiring dose interruption or reduction.

\section{Case 2}

A 79-year-old non-Hispanic white male was diagnosed with ITP 8 years ago and had been managed intermittently with corticosteroids. He was most recently referred to a hematologist when his cardiologist requested an opinion, given the patient's anxiety about 
Table 1. Case characteristics

\begin{tabular}{|c|c|c|c|c|c|c|c|}
\hline & $\begin{array}{l}\text { Age, years, } \\
\text { sex }\end{array}$ & Race/ethnicity & $\begin{array}{l}\text { Thrombotic } \\
\text { risk factors }\end{array}$ & $\begin{array}{l}\text { Year of ITP } \\
\text { diagnosis }\end{array}$ & $\begin{array}{l}\text { Time from diagnosis to start } \\
\text { of fostamatinib, months }\end{array}$ & $\begin{array}{l}\text { Previous ITP } \\
\text { treatments }^{\mathrm{a}}\end{array}$ & $\begin{array}{l}\text { Baseline platelets, } \\
\times 10^{3} / \mu \mathrm{L}\end{array}$ \\
\hline Case 1 & 63 , male & $\begin{array}{l}\text { Non-Hispanic } \\
\text { white }\end{array}$ & $\begin{array}{l}\text { HTN } \\
\text { HLP } \\
\text { T2DM } \\
\text { DVT }\end{array}$ & 2018 & 2 & Dexamethasone & 59 \\
\hline Case 3 & 60 , female & Hispanic & $\begin{array}{l}\text { DVT } \\
\text { Obesity }\end{array}$ & 2015 & $>24$ & $\begin{array}{l}\text { Prednisone } \\
\text { Rituximab } \\
\text { Eltrombopag } \\
\text { Platelet } \\
\text { transfusions } \\
\text { Romiplostim }\end{array}$ & 39 \\
\hline Case 4 & 90 , male & $\begin{array}{l}\text { Non-Hispanic } \\
\text { white }\end{array}$ & $\begin{array}{l}\mathrm{AF} \\
\mathrm{CAD}\end{array}$ & 2014 & $>24$ & Prednisone & 17 \\
\hline Case 5 & 82 , male & $\begin{array}{l}\text { Non-Hispanic } \\
\text { white }\end{array}$ & $\begin{array}{l}\text { AF } \\
\text { CAD } \\
\text { Obesity } \\
\text { Smoker }\end{array}$ & 2018 & 7 & $\begin{array}{l}\text { Platelet transfusio } \\
\text { IVlg Prednisone } \\
\text { Rituximab } \\
\text { Eltrombopag }\end{array}$ & $\mathrm{n} 57$ \\
\hline
\end{tabular}

AF, atrial fibrillation; CAD, coronary artery disease; DVT, deep vein thrombosis; HLP, hyperlipidemia; HTN, hypertension; ITP, immune thrombocytopenia; IVIg, intravenous immunoglobulin; PVD, peripheral vascular disease; T2DM, type 2 diabetes mellitus. ${ }^{\text {TTreatments are }}$ referenced in order of prescribing, but patients may have been on 1 or more treatments on numerous occasions. bPlatelet count was obtained immediately before the start of fostamatinib treatment.

being on anticoagulant therapy for management of his atrial fibrillation, coronary artery disease, and peripheral vascular disease. The patient was initially on rivaroxaban $20 \mathrm{mg}$, which was later changed to apixaban to mitigate the risk of bleeding. After WATCHMAN device placement, anticoagulation was switched to dual antiplatelet therapy with aspirin and clopidogrel. Of significance, his history is notable for hypertension, hyperlipidemia, and type 2 diabetes mellitus. Upon consultation with the hematologist, it was decided to keep the patient off ITP treatment because his platelets were 81,000/ $\mu \mathrm{L}$. Shortly after this consult, the patient's platelets decreased to $49,000 / \mu \mathrm{L}$ and he was started on prednisone therapy. Unfortunately, his blood glucose was very difficult to control, increasing to 500 $\mathrm{mg} / \mathrm{dL}$ during steroid treatment, and a taper was initiated. Platelets ranged between $119,000 / \mu \mathrm{L}$ and $292,000 / \mu \mathrm{L}$ during the taper and blood glucose began to decrease. The patient then remained off therapy for 5 months (platelets $116,000 / \mu \mathrm{L}, 74,000 / \mu \mathrm{L}$ ) until platelets decreased to $22,000 / \mu \mathrm{L}$, and he was started on a lower prednisone regimen with a plan to initiate rituximab for 4 cycles. Blood glucose remained normal, but platelets did not demonstrate a durable response, and the patient developed epistaxis and thrombocytopenia $(36,000 / \mu \mathrm{L})$. His hematologist discussed various longterm treatment options, and it was mutually decided to begin fostamatinib due to its novel mechanism of action and lack of risk for thromboembolism. During the first year of treatment with fostamatinib, the patient's blood glucose remained within normal limits and his platelets were consistently above $100,000 / \mu \mathrm{L}$. The patient experienced a slight increase in blood pressure during the first week of therapy, but this was successfully managed by increasing the dose of his antihypertensive medication. No other adverse effects were reported, and the patient was able to remain on dual antiplatelet therapy for management of his cardiac disease. No fostamatinib dose reductions or omissions have been attempted.

\section{Case 3}

The third patient case is a 60 -year-old Hispanic female with a history of obesity and ITP. She was diagnosed with ITP in 2015, after complaining of fatigue and bilateral lower extremity petechiae. The patient's ITP management was complicated by a slew of adverse effects associated with the various treatments she received. When treated with corticosteroids, the patient complained of insomnia and anxiety. She was treated with 3 doses of rituximab, and although her platelet response was adequate $(269,000 / \mu \mathrm{L})$, the patient experienced rigors and nausea during infusions. She subsequently developed a DVT in her left leg that she attributed to treatment with rituximab; thus, she refused further doses. The patient's DVT was managed with rivaroxaban; she was then started on a 


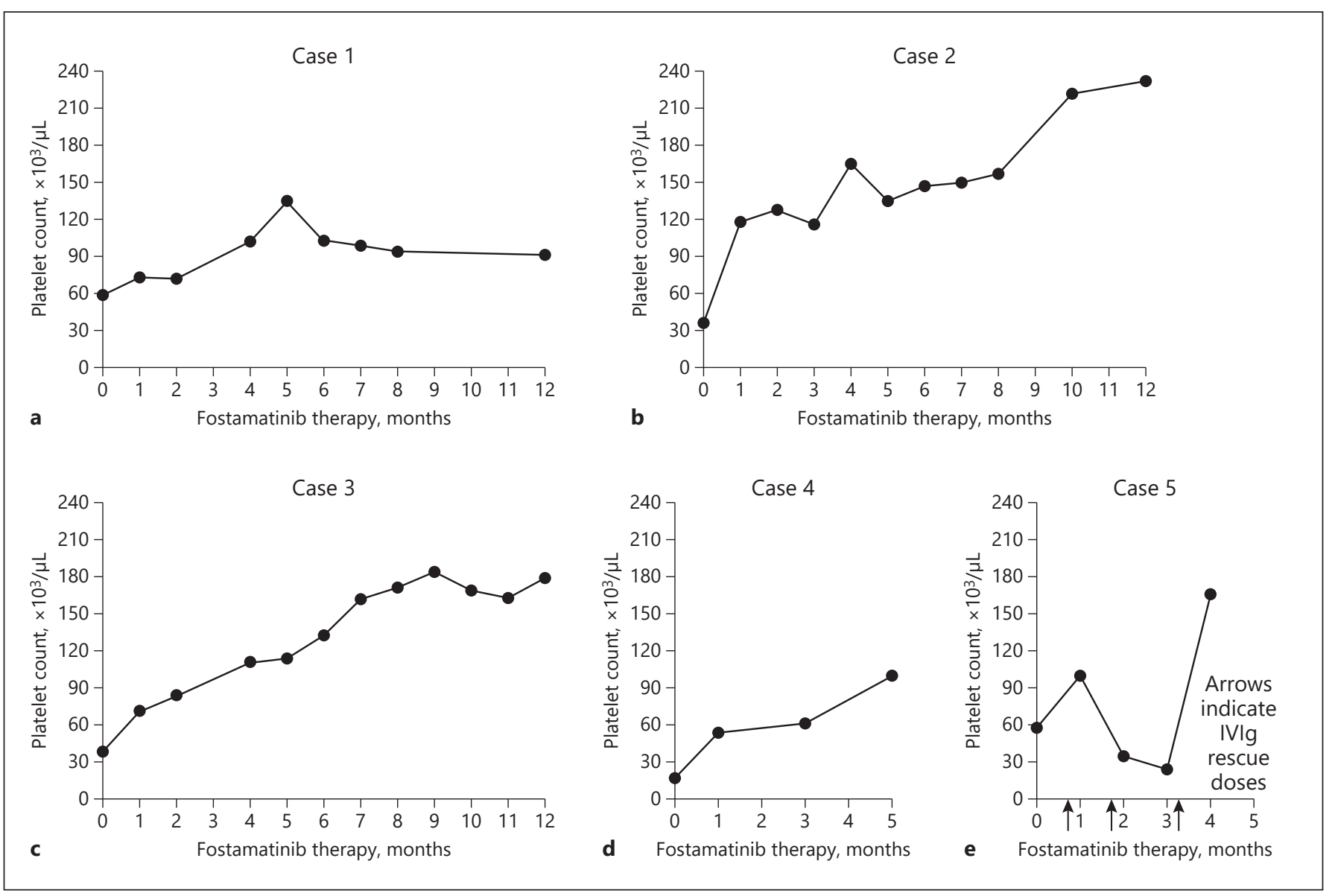

Fig. 1. Platelet response over months of fostamatinib therapy. Patients in a-c (cases 1-3) were managed with fostamatinib for at least 1 year, with $\geq 8$ months of platelet counts available within that first year. Three months of platelet counts were available for the patient in $\mathbf{d}$ (case 4), and 4 months for the patient in e (case 5), during which he received rescue therapy with IVIg in months 1, 2, and 3. BL, baseline; IVIg, intravenous immunoglobulin.

short course of eltrombopag, which caused her to develop headaches and to self-discontinue treatment. To manage her consistently low platelet counts $(10,000-20,000 / \mu \mathrm{L})$, the patient received intermittent platelet transfusions, and after about 8 months, it was decided to start her on romiplostim injections. After 2 doses, her platelets increased to $810,000 / \mu \mathrm{L}$ and the patient developed a second DVT, for which she was again prescribed rivaroxaban. She was referred to another hematologist for a second opinion, and it was decided to start her on fostamatinib $100 \mathrm{mg}$ PO BID due to low thrombotic risk, added benefit of oral administration, a steady platelet response, and a tolerable side effect profile. Once on treatment, the patient no longer had signs of bleeding or bruising, and within 3 months her platelets increased from $39,000 / \mu \mathrm{L}$ to consistently above $100,000 / \mu \mathrm{L}$. She has now been on fostamatinib for almost 2 years (platelet range 72,000-199,000/ $\mu \mathrm{L}$ ) and has not experienced any additional side effects, including no DVT recurrence. No fostamatinib dose reductions or omissions were attempted during the reporting period.
Case 4

A 90-year-old non-Hispanic white male was diagnosed with ITP 6 years ago. He was on anticoagulation with apixaban for a history of atrial fibrillation and coronary artery disease with coronary artery bypass graft. He was not on any treatment for the chronic ITP, but his platelets began to progressively decline through the years, and he developed an upper gastrointestinal bleed due to a Mallory-Weiss tear. At the time, the patient's platelet count was $34,000 / \mu \mathrm{L}$ and prednisone was started. Apixaban was discontinued and platelets initially responded, increasing to $51,000 / \mu \mathrm{L}$. Two weeks later, platelets decreased to $17,000 / \mu \mathrm{L}$. The patient was reluctant to be on long-term corticosteroid therapy; after discussing various options with his physician, a prednisone taper was started and the patient decided to begin fostamatinib at $100 \mathrm{mg}$ PO BID. He tolerated fostamatinib well, with no reports of adverse effects. His immature platelet fraction and mean platelet volume both decreased steadily after the start of fostamatinib treatment, coinciding with an increase in platelet count. His platelets 
have remained $>90,000 / \mu \mathrm{L}$ and he has been able to start aspirin for atrial fibrillation thromboprophylaxis. No fostamatinib dose changes or omissions were attempted.

\section{Case 5}

An 82-year-old non-Hispanic white male was diagnosed with ITP after he noticed new-onset petechiae, epistaxis, and some oral mucosal bleeding. He was seen in the emergency department, where his platelets were noted to be $\langle 5,000 / \mu \mathrm{L}$; he was treated with platelet transfusion and 1 dose of IVIg and commenced on prednisone $60 \mathrm{mg}$ PO once-daily (QD). Despite a history of atrial fibrillation, hypertension, coronary artery disease with angioplasty, obesity, and smoking, low-dose aspirin was discontinued due to thrombocytopenia. Platelets responded to corticosteroids for about a month, and the patient did not complain of any side effects. Platelets dropped again to $<10,000 / \mu \mathrm{L}$, and the patient was started on weekly rituximab infusions for 6 weeks. The response to rituximab was not durable, and consequently, other treatment options were discussed. Fostamatinib was the provider's preferred choice to avoid the risks of thromboembolism from other alternatives, but it was not covered by the patient's insurance. The patient was started on eltrombopag. During therapy, his platelet counts waxed and waned, and he required intermittent IVIg therapy. After 4 months of eltrombopag, the patient's insurance company approved fostamatinib, and he was started on $100 \mathrm{mg}$ PO BID. The dose was increased to $150 \mathrm{mg}$ PO BID after 4 weeks, and the patient received IVIg on 3 more occasions. Although the platelet response was initially slow and he required rescue therapy with IVIg, his most recent platelet count was $105,000 / \mu \mathrm{L}$. Further, he was noted to maintain a longer response without use of rescue while on fostamatinib than with eltrombopag. Since the start of fostamatinib treatment, the patient has had 1 episode of diarrhea, which was self-limiting, and his blood pressure has been well controlled. No further changes to the fostamatinib dose occurred during the reporting period.

\section{Discussion}

The 5 cases in this report highlight the challenges associated with selecting the best treatment for ITP and successfully managing patients. Although these patients had similarities in their medical history, specifically CV disease and/or thrombotic risk factors with need for ongoing anticoagulant or antiplatelet therapy, the presentation and course of therapy for ITP were unique in each case. Patients in this case series had approximately 23 cumulative patient-years of history of ITP. Two patients had persistent ITP (cases 1 and 5), and 3 patients had chronic ITP (cases 2, 3, and 4). The number of previous treatments ranged from 1 to 5 medications, including corticosteroids for all 5 patients, rituximab for 3 patients, and platelet transfusions and TPO-RAs for 2 patients. Fostamatinib was introduced as a second-line agent for 2 patients but as a third-line or later agent for the other 3 patients. Despite the heterogeneity of ITP in these 5 cases, the patients in this case series were all successfully treated with fostamatinib without further thromboembolic events.

Three patients have now been taking fostamatinib for longer than 1 year. Four patients had a rapid platelet response, with platelet counts increasing and remaining above $50,000 / \mu \mathrm{L}$ during the first month of treatment. It is important to note that there was no evidence of bleeding or bruising while patients were taking fostamatinib, and only case 5 required the use of rescue therapy during treatment. This durability of response is not surprising, as the published post hoc analysis of patients from clinical studies showed a robust response, with platelets $\geq 50,000 /$ $\mu \mathrm{L}$ maintained at a median of $83 \%$ and $86 \%$ of treatment days for patients who received fostamatinib as a secondline or third-line or later agent, respectively [24]. In this case series, no patient experienced abnormally high platelet counts while taking fostamatinib, in contrast to this concern when patients are treated with TPO-RAs. As is well documented, the mechanism of action of TPO-RAs is to increase the platelet count by stimulating megakaryocyte function within the bone marrow [25]. Further, evidence suggests that newly produced immature platelets, such as those produced when TPO-RAs are taken, are more hemostatically active than mature platelets and may contribute to thrombotic events [26]. Platelet counts $>200,000 / \mu \mathrm{L}$ have been associated with high discontinuation rates in some clinical studies and have coincided with thrombotic adverse events in some patients, as noted in case 3 when managed with romiplostim [27-30]. Although platelet count may not necessarily trigger thrombus formation, avoiding overcorrection of platelet counts remains a relevant consideration for management of ITP [2, 30-32].

As in the clinical studies, fostamatinib was well tolerated by all 5 patients in this series, with only 1 occurrence each of mild diarrhea and mild hypertension; hypertension was managed with a dose increase of antihypertensive medication. This safety profile differs substantially from that seen with other second-line agents used to manage ITP. Rituximab, an anti-CD20 monoclonal antibody that is not FDA-approved for management of ITP, may cause infusion reactions (as seen in case 2) and carries a black box warning for reactivation of hepatitis B virus, severe mucocutaneous reactions, and the rare occurrence of progressive multifocal leukoencephalopathy $[1,3]$. Splenectomy has fallen out of favor due to high relapse rates, lifelong increased risk of infection and sepsis, and increasing numbers of other available medical agents for ITP; it is noteworthy that splenectomy conveys an associated risk for thrombosis, as is seen with TPO-RAs $[2,8]$. The an- 
nualized rate of thrombosis with TPO-RAs is 2-3 times higher than in an ITP population not treated with TPORAs $[6,30,31]$, and a recent report of ITP patients taking long-term fostamatinib noted a rate of $<1 \%$ [33].

Although the 5 patients in this case series had underlying $\mathrm{CV}$ and/or thromboembolic risk factors, it is important to mention that ITP itself may paradoxically increase the risk for thromboembolic events. Arterial and venous thrombosis in patients with ITP is thought to be due, at least in part, to activation of platelets by autoantibodies and the fact that ITP is an inherently procoagulant condition, as indicated by an increase in various coagulation activation markers $[2,31]$. There is further laboratory evidence for procoagulant pathophysiology in ITP as well, including demonstrated higher in vivo levels of prothrombin fragments $1+2, \mathrm{D}$-dimer, and plasminogen activator inhibitor-1 (PAI-1), all of which contribute to thrombophilic risk [25]. It is intriguing that upon treatment with TPO-RAs, this procoagulant profile was accentuated toward greater thrombogenicity by further increase in PAI-1 and soluble P-selectin [25]. This is additionally supported by a study of 82 chronic ITP patients, in which patients treated with TPO-RAs had significantly increased PAI-1 levels compared to controls [34]. Clinical concern for thromboembolic risk has been made even more apparent during the COVID-19 pandemic, as increased inflammatory markers and interleukins in SARS$\mathrm{CoV}$-2-positive patients have been noted to stimulate the coagulation cascade and create a hypercoagulable state, even in patients with thrombocytopenia $[35,36]$.

Avoiding medications that heighten the prothrombotic state (e.g., TPO-RAs) or exacerbate underlying conditions (e.g., corticosteroids) is critical, as increased risk for morbidity and mortality has been observed in ITP patients with CV disease. In a population-based cohort of adults with ITP, the adjusted hazard ratio for mortality due to CV disease was 1.5 (95\% confidence interval [CI], 1.1-1.5) [37]. A retrospective cohort study in ITP patients found that age $>60$ years, steroid use, and $>2$ risk factors for thrombosis were independently associated with increased risk of thrombosis $[8,32]$. All patients in this case series met these criteria, but 4 out of 5 were able to continue their cardioprotective antiplatelet or anticoagulant medication while keeping other underlying CV and/or thrombotic comorbidities well controlled during therapy with fostamatinib. Additionally, none of the patients in this series required rescue with corticosteroids, thereby reducing the likelihood of exacerbating $\mathrm{CV}$ risk factors (e.g., diabetes and hypertension). It is imperative to identify patients with comorbidities that further increase their risk of thromboembolism and to ensure that selected treatments do not worsen underlying disease states or increase the likelihood of thrombosis [7].

A limitation of this case series report is the retrospective nature of identifying cases with chronic ITP and high risk for thrombosis due to $\mathrm{CV}$ comorbidities or history of thrombosis. Furthermore, not all such clinical scenarios confer equal thrombophilic risk. However, due to the unpredictable frequency by which such scenarios present in practice, and the clinical dilemma that concurrent thrombophilic conditions with severe thrombocytopenia pose, we felt that retrospective identification of representative cases from hematological practice was appropriate.

\section{Conclusion}

Overall, ITP necessitates careful consideration of multiple competing clinical factors when the most appropriate agent is selected for patient treatment. For patients with clot-provoking disease, it is evident that the choice may be even more challenging, and it is important that clinicians carefully evaluate the patient's medical history and the safety profile of the various treatment options [2]. Involving patients in the decision-making process may contribute to a more successful treatment plan. In this case series of patients with multiple underlying CV and/ or thrombotic risk factors, fostamatinib was observed to be a safe and reliable treatment for the management of ITP. Successfully and safely treating patients with ITP who are at high risk for thromboembolic and CV events present an important patient dilemma, and publications highlighting real-world experience may be useful to clinicians when selecting therapies for these patients.

\section{Acknowledgments}

We thank the patients and their families for participating in this case series. The authors thank Sandra M. Aguero, PharmD, BCPS, and Dolores Matthews, ELS, of PharmaWrite, LLC, for medical writing and editorial assistance, which were funded by Rigel Pharmaceuticals, Inc.

\section{Statement of Ethics}

These cases reflect the clinical observations reported from 5 retrospective cases using de-identified data, and Institutional Review Board approval was not required. Written informed consent was obtained from all patients for publication of this case series. 


\section{Conflict of Interest Statement}

A.M. and A.K. have received consultant fees and/or honoraria from Rigel Pharmaceuticals. C.T. has received speaker honoraria from Rigel Pharmaceuticals. D.S. has no conflicts of interest to declare.

\section{Funding Sources}

Drs. Yuliya Kreychman and Aaron Sheppard, of Rigel Pharmaceuticals, Inc., were involved in case discussion with the authors and manuscript preparation in an advisory board setting. In accordance with ICMJE authorship criteria and GPP3 guidelines, the authors received professional medical writing assistance provided by Sandra M. Aguero, PharmD, BCPS, and editorial support from Dolores Matthews, ELS, of PharmaWrite, LLC, which were funded by Rigel Pharmaceuticals, Inc.

\section{Author Contributions}

All authors contributed equally and all were involved in critically revising the manuscript. All authors reviewed the final manuscript and gave approval for submission.

\section{Data Availability Statement}

All data generated or analyzed during this study are included in the article. Further inquiries can be directed to the corresponding author.

\section{References}

1 Lakshmanan S, Cuker A. Contemporary management of primary immune thrombocytopenia in adults. J Thromb Haemost. 2012; 10:1988-98.

2 Moulis G, Comont T, Adoue D. [New insights into the epidemiology of immune thrombocytopenia in adult patients: impact for clinical practice]. Rev Med Interne. 2021;42(1):11-5.

3 Provan D, Arnold DM, Bussel JB, Chong BH, Cooper N, Gernsheimer T, et al. Updated international consensus report on the investigation and management of primary immune thrombocytopenia. Blood Adv. 2019;3:3780817.

4 Neunert C, Terrell DR, Arnold DM, Buchanan G, Cines DB, Cooper N, et al. American Society of Hematology 2019 guidelines for immune thrombocytopenia. Blood Adv. 2019:3:3829-66.

5 Garabet L, Henriksson CE, Lozano ML, Ghanima W, Bussel J, Brodin E, et al. Markers of endothelial cell activation and neutrophil extracellular traps are elevated in immune thrombocytopenia but are not enhanced by thrombopoietin receptor agonists. Thromb Res. 2020;185:119-24.

6 van Dijk WEM, Brandwijk ON, Heitink-Polle KMJ, Schutgens REG, van Galen KPM, Urbanus RT. Hemostatic changes by thrombopoietin-receptor agonists in immune thrombocytopenia patients. Blood Rev. 2020;47: 100774.

7 Ekstrand C, Linder M, Baricault B, Lafaurie M, Sailler L, Lapeyre-Mestre M, et al. Impact of risk factors on the occurrence of arterial thrombosis and venous thromboembolism in adults with primary immune thrombocytopenia: results from two nationwide cohorts. Thromb Res. 2019;178:124-31.

8 Rodeghiero F. Is ITP a thrombophilic disorder? Am J Hematol. 2016;91:39-45.
9 Balitsky AK, Kelton JG, Arnold DM. Managing antithrombotic therapy in immune thrombocytopenia: development of the TH2 risk assessment score. Blood. 2018;132:26846.

10 Newland A, Lee EJ, McDonald V, Bussel JB. Fostamatinib for persistent/chronic adult immune thrombocytopenia. Immunotherapy. 2018;10:9-25.

11 Pamuk ON, Tsokos GC. Spleen tyrosine kinase inhibition in the treatment of autoimmune, allergic and autoinflammatory diseases. Arthritis Res Ther. 2010;12:222.

12 Niscola P, Scaramucci L, Giovannini M. Spleen tyrosine kinase inhibition: a new promising approach to chronic and refractory immune thrombocytopenia. Immunotherapy. 2018;10:5-7.

13 Podolanczuk A, Lazarus AH, Crow AR, Bussel JB. Of mice and men: an open-label pilot study for treatment of immune thrombocytopenic purpura by an inhibitor of Syk. Blood. 2009; 113:3154-60.

14 Andre P, Morooka T, Sim D, Abe K, Lowell C, Nanda N, et al. Critical role for Syk in responses to vascular injury. Blood. 2011;118: 5000-10.

15 Manne BK, Badolia R, Dangelmaier C, Eble JA, Ellmeier W, Kahn M, et al. Distinct pathways regulate Syk protein activation downstream of immune tyrosine activation motif (ITAM) and hemITAM receptors in platelets. J Biol Chem. 2015;290:11557-68.

16 Matus V, Valenzuela G, Sáez CG, Hidalgo P, Lagos M, Aranda E, et al. An adenine insertion in exon 6 of human GP6 generates a truncated protein associated with a bleeding disorder in four Chilean families. J Thromb Haemost. 2013;11:1751-9.
17 Payne H, Ponomaryov T, Watson SP, Brill A. Mice with a deficiency in CLEC-2 are protected against deep vein thrombosis. Blood. 2017; 129:2013-20.

18 van Eeuwijk JMM, Stegner D, Lamb DJ, Kraft $\mathrm{P}$, Beck S, Thielmann I, et al. The novel oral syk inhibitor, Bl1002494, protects mice from arterial thrombosis and thromboinflammatory brain infarction. Arterioscler Thromb Vasc Biol. 2016;36:1247-53.

19 Xia L. Platelet CLEC-2: a molecule with 2 faces. Blood. 2017;130:2158-60.

20 Spalton JC, Mori J, Pollitt AY, Hughes CE, Eble JA, Watson SP. The novel Syk inhibitor R406 reveals mechanistic differences in the initiation of GPVI and CLEC-2 signaling in platelets. J Thromb Haemost. 2009;7:1192-9.

21 Stegner D, Haining EJ, Nieswandt B. Targeting glycoprotein VI and the immunoreceptor tyrosine-based activation motif signaling pathway. Arterioscler Thromb Vasc Biol. 2014;34:1615-20.

22 Bussel J, Arnold DM, Grossbard E, Mayer J, Treliński J, Homenda W, et al. Fostamatinib for the treatment of adult persistent and chronic immune thrombocytopenia: results of two phase 3, randomized, placebo-controlled trials. Am J Hematol. 2018;93:92130.

23 Bussel JB, Arnold DM, Boxer MA, Cooper N, Mayer J, Zayed H, et al. Long-term fostamatinib treatment of adults with immune thrombocytopenia during the phase 3 clinical trial program. Am J Hematol. 2019;94:546-53.

24 Boccia R, Cooper N, Ghanima W, Boxer MA, Hill QA, Sholzberg M, et al. Fostamatinib is an effective second-line therapy in patients with immune thrombocytopenia. Br J Haematol. 2020;190:933-8. 
25 Garabet L, Ghanima W, Monceyron Jonassen C, Skov V, Holst R, Mowinckel MC, et al. Effect of thrombopoietin receptor agonists on markers of coagulation and $\mathrm{P}$-selectin in patients with immune thrombocytopenia. Platelets. 2019;30:206-12.

26 Grove EL, Hvas AM, Kristensen SD. Immature platelets in patients with acute coronary syndromes. Thromb Haemost. 2009; 101: 151-6.

27 Bussel JB, Cheng G, Saleh MN, Psaila B, Kovaleva L, Meddeb B, et al. Eltrombopag for the treatment of chronic idiopathic thrombocytopenic purpura. N Engl J Med. 2007;357: 2237-47.

28 Bussel JB, Kuter DJ, Aledort LM, Kessler CM, Cuker A, Pendergrass KB, et al. A randomized trial of avatrombopag, an investigational thrombopoietin-receptor agonist, in persistent and chronic immune thrombocytopenia. Blood. 2014;123:3887-94.

29 Bussel JB, Provan D, Shamsi T, Cheng G, Psaila B, Kovaleva L, et al. Effect of eltrombopag on platelet counts and bleeding during treatment of chronic idiopathic thrombocytopenic purpura: a randomised, doubleblind, placebo-controlled trial. Lancet. 2009; 373:641-8.

30 Kuter DJ, Bussel JB, Newland A, Baker RI, Lyons RM, Wasser J, et al. Long-term treatment with romiplostim in patients with chronic immune thrombocytopenia: safety and efficacy. Br J Haematol. 2013;161:411-23.

31 Ghanima W, Cooper N, Rodeghiero F, Godeau B, Bussel JB. Thrombopoietin receptor agonists: ten years later. Haematologica. 2019;104:1112-23.

32 Ruggeri M, Tosetto A, Palandri F, Polverelli N, Mazzucconi MG, Santoro C, et al. Thrombotic risk in patients with primary immune thrombocytopenia is only mildly increased and explained by personal and treatment-related risk factors. J Thromb Haemost. 2014; 12:1266-73.

33 Cooper N, Altomare I, Thomas MR, Nicolson PLR, Watson SP, Markovtsov V, et al. Assessment of thrombotic risk during long-term treatment of immune thrombocytopenia with fostamatinib. Ther Adv Hematol. 2021;12: 20406207211010875.

34 Justo Sanz R, Monzón Manzano E, Fernández Bello I, Teresa Álvarez Román M, Martín Salces M, Rivas Pollmar MI, et al. Platelet apoptosis and pai-1 are involved in the pro-coagulant state of immune thrombocytopaenia patients treated with thrombopoietin receptor agonists. Thromb Haemost. 2019;119: 645-59.

35 Levi M, Thachil J, Iba T, Levy JH. Coagulation abnormalities and thrombosis in patients with COVID-19. Lancet Haematol. 2020;7: e438-40.

36 Nicolson PL, Welsh JD, Chauhan A, Thomas MR, Kahn ML, Watson SP. A rationale for blocking thromboinflammation in COVID-19 with Btk inhibitors. Platelets. 2020; 31:685-90.

37 Frederiksen H, Maegbaek ML, Nørgaard M. Twenty-year mortality of adult patients with primary immune thrombocytopenia: a Danish population-based cohort study. Br J Haematol. 2014;166:260-7. 